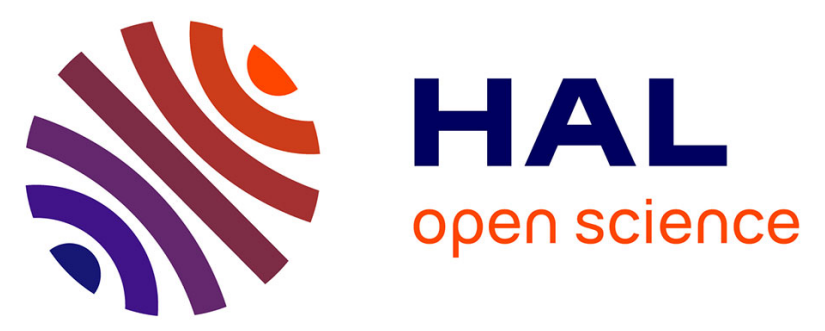

\title{
A large thermal turbulent Taylor-Couette (THETACO) facility for investigation of turbulence induced by simultaneous action of rotation and radial temperature gradient
}

Harminder Singh, Antoine Bonnesoeur, Hugues Besnard, Claude Houssin, Arnaud Prigent, Olivier Crumeyrolle, Innocent Mutabazi

\section{To cite this version:}

Harminder Singh, Antoine Bonnesoeur, Hugues Besnard, Claude Houssin, Arnaud Prigent, et al.. A large thermal turbulent Taylor-Couette (THETACO) facility for investigation of turbulence induced by simultaneous action of rotation and radial temperature gradient. Review of Scientific Instruments, 2019, 90 (11), pp.115112. 10.1063/1.5119811 . hal-03236921

\section{HAL Id: hal-03236921}

https://hal-normandie-univ.archives-ouvertes.fr/hal-03236921

Submitted on 26 May 2021

HAL is a multi-disciplinary open access archive for the deposit and dissemination of scientific research documents, whether they are published or not. The documents may come from teaching and research institutions in France or abroad, or from public or private research centers.
L'archive ouverte pluridisciplinaire HAL, est destinée au dépôt et à la diffusion de documents scientifiques de niveau recherche, publiés ou non, émanant des établissements d'enseignement et de recherche français ou étrangers, des laboratoires publics ou privés. 


\title{
A large Thermal Turbulent Taylor-Couette (THETACO) facility for investigation of turbulence induced by simultaneous action of rotation and radial temperature gradient
}

\author{
Harminder Singh, Antoine Bonnesoeur, Hugues Besnard, Claude Houssin, Arnaud Prigent ${ }^{1}$, \\ Olivier Crumeyrolle and Innocent Mutabazi
}

\begin{abstract}
Normandie Université, UNIHAVRE, CNRS, Laboratoire Ondes et Milieux Complexes (LOMC), UMR 6294, 53 Rue de Prony, Le Havre 76058 Cedex, France
\end{abstract}

\begin{abstract}
A thermal Taylor-Couette facility (THETACO) has been designed to investigate turbulent flows generated by differential rotation and radial temperature gradient. It consists of a cylindrical annulus with a rotating inner cylinder and fixed outer cylinder. The electric heating system is installed inside the inner cylinder and the annulus is immersed in a large cylindrical container filled with cooling fluid. Temperature regulators control independently the temperature of the inner surface of the inner cylinder and that of the cooling fluid. The facility allows to reach values of the Reynolds number $\left(R e \sim 5 \times 10^{5}\right)$ and of the Rayleigh number $\left(R a \sim 3 \times 10^{6}\right)$ for water as working fluid. The facility provides torque measurements, a full optical access at the side and from the bottom for velocity measurements using PIV (2D, stereoscopic and tomographic). Temperature measurements in the flow can be performed by thermochromic liquid crystals or laser induced fluorescence.
\end{abstract}

\section{INTRODUCTION}

The Taylor-Couette (TC) system, consisting of fluid confined between two concentric differentially rotating cylinders, together with the Rayleigh-Bénard convection (RBC) between two parallel plates with differential heating represents a canonic closed flow to investigate the transition to turbulence ${ }^{1}$. Like the RBC, the TC system has a simple geometry with multiple symmetries, breaking of which leads to different transitions. The driving mechanism of the first instability is the centrifugal force which unbalances the radial pressure gradient and viscous dissipation. Higher instability modes and turbulent flows in the TC flow involve diverse mechanisms such Kelvin-Helmholtz instability, streaks, boundary layer instabilities near the cylindrical walls, ... Some of its states can be accessed either analytically or numerically and experimentally with high precision. For that reason, it has become the hydrogen atom or drosophylae of the fluid dynamics ${ }^{2}$ with a large variety of applications in diverse disciplines:

\footnotetext{
${ }^{1}$ arnaud.prigent@univ-lehavre.fr
} 
chemical engineering $^{3,4}$, fluid mechanics ${ }^{1}$ 5, 6, electrohydrodynamics ${ }^{7}$, biotechnology ${ }^{8}$, astrophysics $^{9-11}$, and soft matter ${ }^{2}$. Turbulence remains one of the unsolved scientific problems of contemporary Physics and the Taylor-Couette flow is one of the simple hydrodynamic systems which allows a detailed investigation of the transition to turbulence. In the last decade, new experimental Taylor-Couette facilities (with either rotating inner cylinder or both in corotation or in counter-rotation) have been designed to investigate the ultimate regime of turbulence [Twente, Cottbus, ...] which occurs when the boundary layer near cylindrical surface becomes turbulent ${ }^{12-20}$. Table 1 presents features of the known Taylor-Couette geometries around the world.

The ultimate regime of turbulence has also been investigated in the last decade in the case of RBC, where the driving force of the flow is the buoyancy due to the temperature difference between the top and bottom plates ${ }^{21-30}$. Turbulent thermal convection occurs in many processes, such as atmospheric and oceanic circulations, liquid inner core or mantle of planets, transport in accretion disks, and heat exchangers in diverse industrial processes ${ }^{26}$. In many of these processes, the temperature gradient is combined with differential rotation. This is the case in geophysical and astrophysical flows but also in processes such as drilling or cooling of rotating machinery ${ }^{31,32}$, high speed pumps $^{33}$ and solidification of pure metals ${ }^{34}$. Such flow systems can be modelled by a vertical Taylor-Couette flow with a radial temperature gradient (RTG). As soon as a radial temperature gradient is imposed to a fluid under the gravity, it induces a baroclinic flow consisting of a large convection cell with ascending flow near the hot surface and descending near the cold one. As the temperature gradient increases, instability develops in the baroclinic flow creating smaller convective cells within the large convection cell $^{35}$. Combination of rotation and radial temperature gradient in the Taylor-Couette flow leads to a double control parameter system where one can vary independently either the temperature difference or the rotation of the inner cylinder ${ }^{36}$. Variation of these two parameters leads to a rich variety of flow regimes which deserve a thorough investigation.

Snyder and Karlsson ${ }^{37}$ were the first to consider the vertical Taylor-Couette with inner cylinder rotation and a RTG. They observed significant effect of the RTG on the critical Taylor number and critical wavelength even with relatively small RTG. This result was confirmed by Sorour and Coney ${ }^{38}$. In the case of horizontal TC systems free convection is weak and the effect of RTG was found to be negligible on the flow pattern and the vortex spacing ${ }^{39-41}$. In the absence of gravity, the RTG was also found to have negligible impact on the stability curve and the structure of cells. Chen and $\mathrm{Kuo}^{42}$ proved through their theoretical examination the importance 
of gravitational and centrifugal potential and buoyancy to alter, significantly, the stability properties in a TC system with-inner cylinder rotation and a RTG.

For a given Taylor-Couette system (i.e. a fixed gap of width $d$ and length $\mathrm{L}$ together with an inner cylinder radius $r_{i}$ ) and a chosen fluid (of kinematic viscosity $v$, thermal expansion $\alpha$ and thermal diffusivity $\kappa$ ), the flow dynamics is described by three control parameters: the Prandtl number $\operatorname{Pr}=v / \kappa$, the Rayleigh number $R a=\alpha g \Delta T d^{3} /(v \kappa)$ and the Reynolds number $R e=\omega r_{i} d / \nu$. Here $g, \Delta T$ and $\omega$ represent the acceleration due to gravity, the effective temperature difference imposed on the fluid and the angular frequency of the inner cylinder, respectively. The flow configuration itself is characterized by geometrical control parameters (i.e. the radius ratio $\eta=r_{i} /\left(r_{i}+d\right)$ and the aspect ratio $\left.\Gamma=L / d\right)$ and the Froude number $F r=v / \sqrt{g d^{3}}$. Yoshikawa et al. ${ }^{43}$ have shown the sensitivity of the onset of thermoconvection in the Taylor-Couette system with RTG on the Froude number. The Prandtl number represents the diffusive nature of the working fluid, the Rayleigh number is the ratio between the product of diffusive time scales and the square of buoyancy time scale, and the Reynolds number determines the ratio between the viscous time scale and the inertial time scale. Although the Prandtl number is temperature-dependent, it is assumed fixed in a given run of an experiment, if the reference temperature does not vary strongly. The Rayleigh and Reynolds numbers are varied independently. As both parameters are related to destabilization mechanism, one can estimate their relative importance by defining their ratio known as Richardson number $R i=$ $R a /\left(\operatorname{Pr} R e^{2}\right)$. Thermal effects dominate for $R i>1$ and rotation effects dominate for $R i<1$. Additionally, to take into account the flow curvature, the Taylor number is often used instead of $R e$. It is related to Reynolds number as follows: $T a=\left[(1+\eta) / 2 \eta^{2 / 3}\right]^{6} R e^{2}$.

Table 1 shows that there is not any major TC system that can work with RTG currently in a large range of control parameters values apart from the TC system that is presented in this work. Until now, the studies have been conducted in the laboratory scale equipment with access to limited values of $R a$ and $R e$. Ball et al. ${ }^{44}$ studied a Rayleigh number of $10^{4}$, Liu et al ${ }^{45}$ reached a Rayleigh number of $6.2 \times 10^{3}$ and are currently upgrading their equipment to be able to study a higher range ${ }^{45}$. More recently Guillerm et al. ${ }^{36}$ presented their work covering a larger range of temperatures leading to $R a \leq 5 \times 10^{3}$.

Apart from the narrow range of experimental data, the amount of research works that have been done in this domain numerically or theoretically is also limited. Most of the theoretical studies of the linear stability analysis and DNS related to this problem have been based on assumptions of Archimedean buoyancy ${ }^{40}$, axisymmetric perturbations ${ }^{42,45}$ or constant 
fluid properties ${ }^{46}$. Recently, Yoshikawa et al. ${ }^{43}$ have presented results from linear stability analysis with a RTG by considering both the centrifugal and Archimedean buoyancies within the Boussinesq approximation for fixed values of $\mathrm{Pr}=5.5$ and $\mathrm{Fr}=7.3 \times 10^{-4}$. They tested the stability of the flow against the axisymmetric and non-axisymmetric perturbations and built bifurcation diagram in the plane spanned by $G r=R a / P r$ and $T a$. It should be noted that Bahloul et al. ${ }^{35}$ had presented earlier a complete linear stability analysis of the non-isothermal flow in a cylindrical annulus without rotation within the Boussinesq approximation; they investigated the effects of the radius ratio and the Prandtl on critical modes.

Experimentally, flow visualization has been the predominant method for investigation of thermoconvective flows in cylindrical annuli with $\mathrm{RTG}^{36-38,48-50}$. More recently, Liu et al ${ }^{51}$ presented a 2D-PIV (Particle Image Velocimetry) study of velocity and vorticity profiles at a maximum Rayleigh number of $3.5 \times 10^{4}$. They ${ }^{51}$ observed an influence of the RTG on the transition process and the critical Reynolds number; however, at large Reynolds number values they found that the transition process was similar to that of an isothermal condition. Nevertheless, there is an extensive amount of experimental work still to be done not only to increase the data range but also to implement new experimental methods, such as Stereo-PIV and Tomographic PIV. The later will allow to capture the three velocity components and their gradients and to provide quantitative characterization of thermoconvective turbulent flows.

Table 1. Comparison of different international Taylor-Couette facilities

\begin{tabular}{|c|c|c|c|c|c|c|c|}
\hline Laboratory & Göttingen $^{52}$ & Twente $^{53}$ & $\begin{array}{l}\text { Boiling } \\
\text { Twente }\end{array}$ & Cottbus $^{55}$ & Maryland $^{56}$ & Princeton $^{57}$ & Le Havre \\
\hline Aspect ratio & Flexible & Flexible & 18.3 & 20 & 69.5 & 2.1 & 48.75 \\
\hline Radius ratio & $\begin{array}{l}0.71 ; 0.88 ; \\
0.98\end{array}$ & $\begin{array}{l}0.716 \quad- \\
0.909\end{array}$ & 0.714 & 0.5 & 0.7245 & 0.348 & 0,869 \\
\hline $\begin{array}{l}\text { Temperature } \\
\text { control }\end{array}$ & $\begin{array}{l}\text { Both } \\
\text { cylinders }\end{array}$ & Only outer & $\begin{array}{l}\text { Both } \\
\text { cylinders }\end{array}$ & $\begin{array}{l}\text { Only } \\
\text { outer }\end{array}$ & Only outer & Unspecified & $\begin{array}{l}\text { Both } \\
\text { cylinders }\end{array}$ \\
\hline $\begin{array}{ll}\text { Reynolds } & \mathrm{N}^{\circ} \\
\text { range } & \end{array}$ & $\sim 10^{6}$ & $\sim 3.4 \times 10^{6}$ & $\sim 4.8 \times 10^{5}$ & $\sim 10^{6}$ & $\sim 4 \times 10^{6}$ & $\sim 10^{7}$ & $\sim 5 \times 10^{5}$ \\
\hline $\begin{array}{l}\text { Rayleigh } \quad \mathrm{N}^{\circ} \\
\text { range }\end{array}$ & NA & NA & NA & NA & NA & NA & $\sim 3.7 \times 10^{6}$ \\
\hline $\begin{array}{l}\text { Torque } \\
\text { measurements }\end{array}$ & On shaft & $\begin{array}{l}\text { In inner } \\
\text { cylinder }\end{array}$ & On shaft & $\begin{array}{l}\text { In inner } \\
\text { cylinder }\end{array}$ & $\begin{array}{l}\text { In inner } \\
\text { cylinder }\end{array}$ & None & On shaft \\
\hline
\end{tabular}




\begin{tabular}{|l|l|l|l|l|l|l|l|}
\hline $\begin{array}{l}\text { Cylinder } \\
\text { rotation }\end{array}$ & Both & Both & Both & Both & Both & Both & Only Inner \\
\hline $\begin{array}{l}\text { Special } \\
\text { features }\end{array}$ & variety in & $\begin{array}{l}\text { Two phase } \\
\text { setup } \\
\text { flow, } \\
\text { variety in } \\
\text { set-up }\end{array}$ & $\begin{array}{l}\text { Boiling } \\
\text { turbulent } \\
\text { flow }\end{array}$ & $\begin{array}{l}\text { Reduced } \\
\text { Ekman } \\
\text { pumping }\end{array}$ & $\begin{array}{l}\text { Very high } \\
\text { Reynolds } \\
\mathrm{N}^{\circ}\end{array}$ & $\begin{array}{l}\text { Negligible } \\
\text { Ekman } \\
\text { pumping, } \\
\text { MRI }\end{array}$ & $\begin{array}{l}\text { Baroclinic } \\
\text { flow, drag } \\
\text { reduction, }\end{array}$ \\
\hline
\end{tabular}

Numerical studies provide an access to the whole flow field for such comprehensive study, as a result, there have been more numerical studies in recent years compared to the experimental ones ${ }^{36,58-60}$. However, numerical studies always need good experimental data to validate their results, especially when the flow becomes more complex and detailed understanding is required. Additionally, numerical studies also suffer from some assumptions to reduce the computational effort, such as periodic boundary conditions, symmetric top and bottom endplates, which would have required no-slip boundary conditions. These assumptions generate certain discrepancies between numerical results and experimental data, which further refers to the importance of experimental methods.

To address all these aspects, we have built a new TC system with a RTG allowing full optical access (axially and horizontally from the bottom plate) as shown in Figure 1. This system is accompanied by all the necessary equipment and material to conduct a multitude of experimental analysis including LDV, LIF, PIV and Stereo-PIV. In this configuration, only inner cylinder can be rotated to a maximum frequency of $30 \mathrm{~Hz}$ while the outer cylinder remains stationary. The RTG is obtained by heating the inner cylinder via an electric coil inside it and cooling the outer one using a water bath connected to a large thermostat.

The paper is organized as follows: the next section describes in detail the THETACO system, and the following two sections provide preliminary results and conclusions.

\section{SYSTEM DESCRIPTION}

\section{A. Generalities}

The THETACO facility consists of two coaxial cylinders mounted on a horizontal system with controlled anti-vibration system. The two coaxial cylinders are of radii $r_{i}=132.5$ $\mathrm{mm}$ and $r_{o}=152.5 \mathrm{~mm}$; the gap filled between the cylinders has a width $d=20 \mathrm{~mm}$ respectively and a working length $L=975 \mathrm{~mm}$ (Figure 2). As such, the dimensionless geometrical parameters are the radius ratio $\eta=r_{i} / r_{o}=0.869$ and the aspect ratio $\Gamma=L / d=$ 48.75. The inner cylinder is connected to a motor and can rotate with the maximum frequency 
$f \leq 30 \mathrm{~Hz}$; the outer cylinder is stationary. The gap is filled with a working fluid of density $\rho$, kinematic viscosity $v$, thermal expansion coefficient $\alpha$ and thermal diffusivity $\kappa$. As mentioned earlier, the physical dimensionless control parameters are the Prandtl number Pr the Froude number $F r$, the Rayleigh number $R a$ and the Reynolds number $R e$.

Figure 1. Schematic drawing of THETACO facility with the lifting system for maintainance, assembly and dismantling of the parts of the equipment. All lengths are given in mm.

The inner cylinder (IC) is made of aluminum alloy 6082 with T6 temper and having black anodized external wall to avoid light reflection and allow a better contrast for flow visualization. This material was chosen for its smooth machinability, excellent corrosion resistance and a good thermal conductivity of $k=172 \mathrm{~W} /(\mathrm{m} . \mathrm{K})$. The outer cylinder (OC) of thickness $e_{o 1}=9 \mathrm{~mm}$ is made of glass to provide full optical access over the entire gap width. Another optical access is also provided on the bottom plate in order to capture the horizontal $r-\theta$ plane for 2D-PIV studies. To control the temperature of the OC, the latter is placed inside another glass cylinder of thickness $e_{o 2}=10 \mathrm{~mm}$ at a gap distance of $37.5 \mathrm{~mm}$ (Figure 2). Water is used as the cooling fluid and is connected to an air-cooled refrigerating unit with a circulation pump of Huber's model unichiller 150T-H.

The top and bottom endplates are fixed along with the stationary outer cylinder. The bottom endplate has a small-area hole underneath from where the gap is filled with the chosen fluid through a reservoir that is placed above the cylinders (Figure 1). This small area on the bottom endplate, can generate some perturbations; however, we have not noticed any significant perturbations on the velocity measurements nor have we observed any significant impact on the flow patterns in the visualizations.

The TC equipment comes with a lifting system that can support up to $250 \mathrm{~kg}$ to facilitate maintenance, assembling and dismantling of the parts of the whole TC equipment. The total weight of the whole system of $1184 \mathrm{~kg}$ is supported on four fixed feet that can be regulated manually to level horizontally. The equipment was designed in such a way that total weight reduces the vibrations produced by the operation of the TC system at high frequency to a negligible level.

The maximum temperature attainable at the inner surface of the inner cylinder is $T_{i}=40^{\circ} \mathrm{C}$ and the lowest temperature of the outer cooling fluid is $T_{o}=10^{\circ} \mathrm{C}$ leading to the maximum temperature difference $\delta T=T_{i}-T_{o}=30^{\circ} \mathrm{C}$. The temperature $T_{i}{ }^{\prime}$ of the outer surface of the 
inner cylinder and the temperature $T_{o}{ }^{\prime}$ of the inner surface of the outer cylinder are given by Equations 1 and 2, respectively:

$$
\begin{aligned}
& T_{i}^{\prime}=T_{i}-R_{a l} \frac{\delta T}{R_{t o t}} \\
& T_{o}^{\prime}=T_{o}+\left(R_{g}+R_{h e}\right) \frac{\delta T}{R_{t o t}}
\end{aligned}
$$

where $R_{a l}=4.4 \times 10^{-5} \mathrm{~K} / \mathrm{W}$ is the resistance due to the inner aluminum cylinder, $R_{g}=$ $1.3 \times 10^{-2} \mathrm{~K} / \mathrm{W}$ is the resistance due to the outer glass cylinder and $R_{h e}=8.2 \times 10^{-4} \mathrm{~K} / \mathrm{W}$ is the resistance due to the forced convection in the fluid on the outer surface of the outer cylinder. The heat convection exchange between cylindrical walls and fluid in the gap is not taken into account, because we wish to use a control parameter that does not depend on the flow regimes. Thus, the resistance due to the fluid within the gap is: $R_{f}=3.8 \times 10^{-2} \mathrm{~K} / \mathrm{W} . R_{t o t}=R_{a l}+$ $R_{f}+R_{h e}+R_{g}$ is the total resistance. Taking into account the thermal resistance of the whole system, the effective temperature difference acting on the fluid in the gap is:

$$
\Delta T=T_{i}^{\prime}-T_{o}^{\prime}=\gamma \delta T=\frac{R_{f}}{R_{t o t}} \delta T
$$

$\gamma$ represents the ratio of fluid resistance to the total resistance $R_{\text {tot }}$. From the values of the different resistances, we obtain the numerical value $\gamma=0.73$ and $\Delta T=22^{\circ} \mathrm{C}$.

Thus, the largest values of the control parameters in the THETACO facility with water as working fluid (fixed values of $\mathrm{Pr}=5.5$ and $\mathrm{Fr}=1.13 \times 10^{-4}$ ) are $R a_{\max }=3.36 \times 10^{6}$ and $R e_{\max }=5 \times 10^{5}$, providing the opportunity to study a large spectrum of flow regimes by varying $R i$.

\section{B. Temperature control}

When working with a radial temperature gradient system or an isothermal system, it is of utmost importance that the consigned value of temperature remains constant and stable because viscosity of most liquids decreases with increase in temperature ${ }^{61}$. In the case of water, the viscosity of water decreases by $\sim 3 \%$ from 5 to $6^{\circ} \mathrm{C}$ and by $\sim 1.8 \%$ from 39 to $40^{\circ} \mathrm{C}$. In the case of more viscous fluid, such as $100 \%$ glycerol, the decrease in the viscosity is more pronounced: $\sim 10.7 \%{ }^{\circ} \mathrm{C}$ from 5 to $6^{\circ} \mathrm{C}$ and by $\sim 6.7 \%$ from 39 to $40^{\circ} \mathrm{C}$. These variations if neglected could impart significant errors in results. In order to maintain a radial temperature gradient at a consigned value, the IC will be heated and maintained at a homogenous temperature axially, to avoid the creation of a forcing vertical temperature gradient, and the OC needs to be cooled at a constant homogenous temperature axially as well. To fulfill these needs, a refrigeration unit is installed around the $\mathrm{OC}$ and a heating unit is installed within the IC. The 
refrigerating unit allows for the control of the outer cooling fluid temperature $T_{o}$ and the heating unit controls $T_{i}$, the temperature of the inner surface of the inner cylinder. Thereby, these two temperatures are considered as fixed in our system.

\section{Refrigerating unit}

The installed refrigerating unit is the model Unichiller 150T-H of the make Huber (Figure 3). It has a cooling power of $15 \mathrm{~kW}$ at $10^{\circ} \mathrm{C}$. It is an air-cooled system which uses the refrigerant R507. This refrigerant is CFC and HCFC free, with an indicated temperature stability of $\pm 0.2{ }^{\circ} \mathrm{C}$ in a temperature range of -20 to $100{ }^{\circ} \mathrm{C}$. It should be noted that a fluctuation of $\pm 0.2{ }^{\circ} \mathrm{C}$ will impact the viscosity of the water by $\sim 0.8 \%$ at around $10^{\circ} \mathrm{C}$ and by $\sim 0.4 \%$ around $39^{\circ} \mathrm{C}$. Moreover, a gradient of $0.2^{\circ} \mathrm{C}$ at $10^{\circ} \mathrm{C}$ generates a variation of Rayleigh number of $\Delta R a \approx 7.87 \times 10^{3}$. Therefore, the fluctuation of $\pm 0.2^{\circ} \mathrm{C}$ is acceptable in the high Rayleigh and Reynolds number turbulent flow. The preliminary tests have shown that the actual fluctuation to maintain the cooling temperature remains within the indicated value of $\pm 0.2{ }^{\circ} \mathrm{C}$.

The refrigerating unit uses self-optimizing adaptive control system to offer a highly precise temperature control. Additionally, it is a high efficiency and eco-friendly system by reducing water consumption and lowering operating costs. Moreover, it provides USB and Ethernet ports for connection with PC or network for remote control or data transmission services. For optimal functioning, it should be operated in ambient temperature range of 5$40^{\circ} \mathrm{C}$.

Figure 2. Fixation system of the three cylinders (inner, intermediate and envelope) and torque meter system. All lengths are given in $\mathrm{mm}$.

\section{Heating unit}

The heating unit is made up of two parts: a) an operational part that consists of the heating elements and the thermometry, as shown in Figure 3; and b) an electrical controlcommand equipment that is integrated into a general electrical cabinet. The operational part is inserted within the hollow IC and it does not touch the IC to avoid interference in the torque measurements. The heating element consists of six stages of infrared toroidal lamps with each one integrated with an infrared pyrometer. These infrared pyrometers of optris Csmicro model $2 \mathrm{~W} \mathrm{LT/LTH}$ can operate within a temperature range of $-40^{\circ} \mathrm{C}$ to $1030^{\circ} \mathrm{C}$ while providing a measurement uncertainty of $\pm 1 \%$ or $\pm 1.0^{\circ} \mathrm{C}$. In order to ascertain the precision of these pyrometers, a preliminary test was conducted in which the temperature was set at $21^{\circ} \mathrm{C}$, and we 
obtained fluctuations of $\pm 0.4{ }^{\circ} \mathrm{C}$ over a period of 600 seconds. These results were $60 \%$ better than the makers' performance indicators. Special care was taken at the time of installation to prevent stray radiation distorting the temperature signal. To maintain homogenous temperature throughout the axial length, a fan is situated at the top of this operational unit to regulate the heat more efficiently. On the other hand, the electrical control-command equipment contains six proportional integral derivative (PID) regulators, one for each heating stage, and enabled the display of these six temperatures on the front of electrical cabinet and control them through these display units. Each heating stage being controlled separately; the system also offers the possibility to study the vertical temperature gradient separately or in conjunction with the radial temperature gradient.

\section{Rotation rate control}

In this system, only the IC can rotate up to a maximum frequency of $30 \mathrm{~Hz}$. It is driven by a direct drive (DDR) servomotor that has a significant advantage over the conventional servomotors due to the elimination of the mechanical transmission by coupling it directly to the load. This aspect improves the reliability, increases its accuracy and repeatability 60 times better than a conventional motor; it offers zero maintenance due to absence of mechanical parts such as belts, gears and gearboxes, and it increases the throughput that was limited by mechanical transmission. Additionally, the DDR servomotor offers audible noise levels as low as $20 \mathrm{dBA}$ and a maximum of $40 \mathrm{dBA}$ at full load, and does not require inertia matching because being directly connected to the load, the load and motor become a common inertia allowing it to run with significantly large inertia ratios, as large as 11000:1.

In order to attain a $30 \mathrm{~Hz}$ rotation rate, the Kollmorgen DDR CH054A electric motor is installed as shown in Figure 4 . It is a powerful three-phase motor of $400 \mathrm{Vac}, 9.82 \mathrm{~A} \mathrm{rms}, 26.5$ A pic, with electric protection of $300 \mathrm{~mA}$ and a maximum speed of $2100 \mathrm{rpm}$. It can achieve a peak torque of $63.8 \mathrm{Nm}$ at peak line current of 26.5A rms. The motor is piloted with the Kollmorgen's workbench 1.13 software which comes with the three-phase Advanced Kollmorgen Drive (AKD) series servo drive having $400 \mathrm{Vac}, 12 \mathrm{~A} \mathrm{rms}, 30 \mathrm{~A}$ pic, $24 \mathrm{~V} \mathrm{dc}, 2 \mathrm{~A}$ with an electric protection of $300 \mathrm{~mA}$. It provides the control of the rotation of the motor in a voltage range of $\pm 10 \mathrm{~V}$ dc. The negative voltage implies that the direction of rotation can be changed. It also allows via two analog outputs the speed of rotation and the torque $(C)$, which it approximates using the formula $T=K \times i$, where $i$ is the current used by the motor and $K$ is the ad-hoc constant which is equal to 2.25 as per the instruction manual. 
Figure 3. (a) The Unichiller 150T-H refrigerating unit. (b) The heating unit inserted in the TC system. All lengths are given in $\mathrm{mm}$.

\section{Torque measurement system}

In order to measure the speed and torque precisely, a mini-smart torque sensor of brand Kistler, and model $4502 \mathrm{~A}$ is interposed between the motor and the drive shaft of the IC. It is powered with a voltage of $24 \mathrm{VDC}$ and delivers a measuring range of 0 to $\pm 5 \mathrm{Vdc}$ corresponding to a torque ranging from 0 to $\pm 50 \mathrm{Nm}$. A $5 \mathrm{~V} \mathrm{dc}$ powered optical encoder delivering 2 times 360 pulses per revolution is integrated in this device to measure rotational speed. It uses strain gage technology with a rigid structure and excellent temperature stability in the range of 10 to $60{ }^{\circ} \mathrm{C}$. It can measure the torque in the range from 0.5 to $50 \mathrm{Nm}$ with an accuracy of $\pm 0.2 \%$.

Figure 4. The electric motor Kollmorgen DDR CH054A and torque sensor Kistler 4502 A directly placed underneath the inner cylinder.

\section{E. Safety features}

Multiple safety features have been put into place in order to prevent certain mechanical problems that may occur during the operation of the TC system:

- To ensure safe operation of the motor, three special features have been put into place. First, in order to absorb large amount energy releases during strong deceleration, a braking resistor is installed with the motor of the make Frizlen having a capacity of 1500 watt of continuous dissipation and $33 \Omega$ of resistance. Secondly, three rollers are placed at $120^{\circ}$ of each other alongside the top the IC to maintain the axe in the vertical position, bringing stability to the motor, and ensuring the rotation of the IC with minimum generation of friction. Finally, three T-type shielded thermocouples are placed inside the rollers, one in each roller, to continuously read the temperatures and in case of an abnormally high temperature, the motor will be brought to a halt automatically.

- Another shielded thermocouple of the T-type is positioned near the point of contact between the movable and stationary portion located at the base and at the center of the inner cylinder to prevent any abnormal heating.

- A lifting system, as shown in Error! Reference source not found., is put into place to safely manage the lifting of heavy pieces of TC system for maintenance, assembly and dismantling of the parts of the system. 
- The whole system can be brought to a halt directly by pressing the emergency stop button, which is present on the electrical cabinet or indirectly through the LabVIEW software. There are specific stop buttons in the case when only a specific equipment needs to be stopped.

- The TC system is placed on the top of a sturdy metal frame to reduce vibration and maintain stability with the whole system weighing in excess of 1.2 tons.

- When laser is in usage, there is a signal located on the front of the laboratory room that indicates that the laser is on and laser specific glasses need to be used in order to enter the room.

\section{F. Instrumentation}

The LabVIEW software in conjunction with the acquisition system Compact RIO 9030 of National Instrument have been installed to manage, capture and control all the necessary information of torque sensor, temperatures within the IC, and rotation of the IC. The Compact Rio 9030 includes an internal memory and a processor to load a program fpga while a second program is already loaded on the pc that allows working in real time. The Compact Rio 9030 is a chassis that can accommodate 4 modules each dedicated to a specific task. In present scenario, the modules that are being used are shown in Figure 5.

1) A TTL module (NI 9401) generating 0-5v signals and also able to read TTL inputs (0-5v) making it possible to generate a voltage pulse (trigger) to trigger an event, for example a pivot acquisition when the cylinder has a very precise position at each cylinder turn. It also allows us to process the signals from the optical encoder to translate them into a rotational speed.

2) $\mathrm{A} \pm 10 \mathrm{~V}$ voltage generation module (NI 9264) is used to generate a voltage that drives the motor speed. This voltage is sent to the drive that supplies the power required by the motor. This module also makes it possible to send the set point temperatures to each PID controller of the heating system in the form of voltages between 0 and $10 \mathrm{~V} \mathrm{dc}$.

3) An analog acquisition module (NI 9205) is used to record the torque, the six voltages from the pyrometers that are then converted into temperature in the LabVIEW software.

4) A temperature acquisition module (NI 9214) is used to record the three temperatures from the rollers, the temperature of the contact point between the base of the cylinder and the TC chassis, as well as a temperature of atmosphere located in the heating system. The latter allows triggering of the ventilation above a set temperature to homogenize the temperature axially in the IC. 


\section{VALIDATION \& CHARACTERIZATION}

In this section, we present preliminary set of results for the 2D-PIV measurements of the mean laminar and turbulent velocity, velocity and temperature measurements for a radial temperature gradient, and torque measurements. In most of the figures presented hereafter, the abscissa is normalized as $x=\left(r-r_{i}\right) / d$, and the ordinate is normalized using * symbol for normalization with $r_{i} \omega=2 \pi f r_{i}$ and + symbol for normalization with $d / v$.

Figure 5. Sketch of the instrumentation for the TC system.

\section{A. Flow visualization}

The flow patterns are visualized with the addition of the small amount of anisotropic particles such as aluminium or Kalliroscope platelets ${ }^{62}$, which can give some information of the flow structures. In our case, the water was mixed with $1 \%$ aqueous suspension of Kalliroscope ST-1000. An example of flow pattern observed in our system is shown in Figure 6 where turbulent longitudinal vortices are spaced along the vertical direction. A laser sheet allows visualizing the cross-section of longitudinal vortices (i.e. the section $r-z$ ). For PIV measurements, instead of Kalliroscope particles, the flow is seeded with hollow spherical glass particles of size $10 \mu \mathrm{m}$.

\section{B. Temperature regimes}

The flow regimes in a cylindrical annulus with an imposed radial temperature gradient depend on the aspect ratio and the radius ratio. A recent numerical study by Lopez at al. ${ }^{63}$ has provided a better insight in the temperature regimes in this flow configuration. They performed multiple numerical simulations within a large range of $\eta(\in[0.1,0.99])$ and $\Gamma(\in[5,80])$. They determined a linear relationship between the Rayleigh number $R a_{*}$ which separates the conduction and the convective regimes, as a function of both the radius $\eta$ and aspect ratios $\Gamma$ : $R a_{*}(\eta, \Gamma)=a(\eta) \Gamma+b(\eta)$ where $a(\eta)=-307.2 \eta^{3}+750.1 \eta^{2}-318.7 \eta+339.6$ while $b$ becomes negligible if $\Gamma \gtrsim 15$. Using their relation for our flow configuration $(\eta=0.869, \Gamma=$ 48.75), we obtain $R a_{*}=2.08 \times 10^{4}$ which corresponds to a temperature difference $\Delta T_{*}=$ $\left(R a_{*} / \operatorname{Pr}\right)\left(v^{2} / \alpha g d^{3}\right)$. Using water as a working fluid, we obtain $\Delta T_{*} \approx 0.17^{\circ} \mathrm{C}$ which is smaller than the temperature precision. Therefore, the laminar flow in our configuration with water is a convective regime. And, to obtain a laminar conductive regime in our configuration we need to use a mixture of glycerol and water with a viscosity of about five times the water viscosity in order to get $\Delta T_{*} \approx 2^{\circ} \mathrm{C}$. 
Figure 6. Visualization of the vertical structures spaced along the axial direction using Kalliroscope platelets and visualization of the meridional cross-section using a laser-sheet for $R e=2.5 \times 10^{4}, R a=0$.

In order to measure the radial temperature profile in the gap, we have seeded in the degasified demineralized water thermochromic liquid crystal (TLC) particles, having the temperature activation range of $20+10^{\circ} \mathrm{C}$. When enlightened by a white light, the color of the light reflected by the TLC particles depends on the temperature. Consequently, when a temperature gradient is established, multiple colors can be observed and measuring the temperature requires a color camera to capture images of the flow. In order to associate the local color to a temperature value, a calibration is performed. The images are captured at multiple isothermal points within the temperature activation range of the TLC particles. Then a Matlab subroutine is then used to extract the radial temperature profile from the captured images using the calibration. In these experiments, a $r-z$ vertical plane was illuminated by a cold light source Schott KL 2500 LCD with a color temperature of $3200 \mathrm{~K}$. The particles in the illuminated plane were captured with a canon EOS-1D mark IV camera with a $100 \mathrm{~mm} \mathrm{f} / 2.8$ objective using $10 \mathrm{fps}$ acquisition frequency. Figure 7 displays the radial temperature profile measured for a temperature difference of $6.6{ }^{\circ} \mathrm{C}$ (with IC at $29{ }^{\circ} \mathrm{C}$ and OC at $20{ }^{\circ} \mathrm{C}$ ) i.e. a Rayleigh number $R a=9.86 \times 10^{5}$. It is similar to the one obtained by Elder ${ }^{64}$ in a rectangular slot for $R a=4.0 \times 10^{5}$.

Figure 7. Radial profile of temperature measured using TLC particles. The temperature of cylindrical surfaces are respectively $T_{i}=29^{\circ} \mathrm{C}$ and $T_{i}{ }^{\prime}=29.0^{\circ} \mathrm{C}$, and $T_{o}=20^{\circ} \mathrm{C}$ and $T_{o}{ }^{\prime}=$ $22.4^{\circ} \mathrm{C} . R a=9.86 \times 10^{5}, R e=0$.

The temperature profile shows a strong decrease near the cylindrical walls $(0<x<0.2$ and $0.8<x<1$ ) where the heat exchange occurs mainly by conduction. Whereas in the bulk zone for $0.2<x<0.8$ the heat exchange occurs via convection.

C. 2D PIV velocity measurements for the temperature gradient

To determine the axial and radial velocity components, 2D-PIV measurements were conducted in the $r-z$ plane. Degasified demineralized water was used as the fluid for 
experiments at a real temperature gradient of $\Delta T=0.73 \times 10=7.3^{\circ} \mathrm{C}$ with $20^{\circ} \mathrm{C}$ at the outer cylinder and $30{ }^{\circ} \mathrm{C}$ at the inner cylinder. Based on the above conditions, the Prandtl number was $\operatorname{Pr}=6.21$ and Rayleigh number, $R a=1.13 \times 10^{6}$. A class four dual power $135-15 \mathrm{Nd}-$ YAG laser was used having $1064 \mathrm{~nm}$ wavelength, 4ns pulse duration and $800 \mathrm{~mJ}$ maximum output. A Hi-Sense CMOS camera of 4MP resolution and $15 \mathrm{fps}$ speed was used with an objective of $50 \mathrm{~mm}$. A total of 1900 dual frame images were recorded for each measurement. The Dantec Dynamics studio version 6.6 was used to compute the vector fields using the adaptive PIV method having the maximum interrogation area size of $32 \times 32$ grid, minimum interrogation area size of $16 \times 16$ grid and grid step size of 4 that gave a spatial resolution of $166.6 \mu \mathrm{m}$.

In Figure 8-a, the mean profile of the axial velocity is shown together with an instantaneous snapshot of the axial velocity (Figure 8-b). Herein, the flow is moving upwards near the hot inner cylinder and downwards near the colder outer cylinder. The instantaneous snapshot of the axial velocity (Figure 8-b) shows the presence of propagating waves in the gap. In Figure 8-a, the difference between the measured axial velocity and the analytical profile of the conduction state, reported in Bahloul et $\mathrm{al}^{35}$ (Equation 4 and 5), is the signature of the presence of the convective regime with wall waves ${ }^{65}$.

$$
\begin{aligned}
& u_{\text {axial }}(r)=\frac{\mathrm{Ra}}{\operatorname{Pr}}\left(C\left(r^{2}-r_{i}^{2}\right)+\left(C\left(r_{o}^{2}-r_{i}^{2}\right)+\frac{1}{4}\left(r_{o}^{2}-r^{2}\right) \frac{\ln \left(r / r_{i}\right)}{\ln \eta}\right)\right) \\
& C=\frac{4 \ln \eta+\left(1-\eta^{2}\right)\left(3-\eta^{2}\right)}{16\left(1-\eta^{2}\right)\left(\left(1+\eta^{2}\right) \ln \eta+1-\eta^{2}\right)}
\end{aligned}
$$

The radial velocity component was found to be 1000 times smaller compared to the axial velocity component. The nodal surface at which the axial flow changes direction in the gap $x_{n}=0.5043$ for the analytical profile and $x_{n}=0.4833$ for the experimental data.

Figure 8. (a) Mean axial velocity at $R a=1.13 \times 10^{6}, R e=0$. (b) Instantaneous snapshot of the axial velocity (given in $\mathrm{m} / \mathrm{s}$ ).

\section{2D-PIV velocity measurements for the IC rotation.}

To determine the azimuthal and radial velocity components, 2D-PIV measurements were done in the $r-\theta$ plane through the transparent bottom plate. A mixture of at $45 \%$ glycerol in water was used as a working fluid for this set of experiments at a fixed temperature of $20^{\circ} \mathrm{C}$. 
The density is $\rho=1128.4 \mathrm{~kg} / \mathrm{m}^{3}$ and the kinematic viscosity is $v=5.5626 \times 10^{-6} \mathrm{~m}^{2} / \mathrm{s}$. The density and kinematic viscosity are estimated based on the parameterization described by Volk and Kähler ${ }^{66}$ which has a maximum deviation of $0.07 \%$ w.r.t measured data. A class four dual power 135-15 Nd-YAG laser was used having $1064 \mathrm{~nm}$ wavelength, $4 \mathrm{~ns}$ pulse duration and $800 \mathrm{~mJ}$ maximum output. An M series speed-sense CMOS camera of 4 MP resolution and 800 fps speed was used with an objective of $105 \mathrm{~mm}$. A total 1350 dual frame images were recorded for each measurement at different rotational speeds. The Dantec Dynamics studio version 6.6 was used to compute the vector fields using the adaptive PIV method having the maximum interrogation area size of $64 \times 64$ grid, minimum interrogation area size of $8 \times 8$ grid and grid step size of 4 that gave a spatial resolution of $56.4 \mu \mathrm{m}$.

Figure 9-a presents a laminar tangential velocity profile at $R e=99.2$ and turbulent mean azimuthal velocity profile at $R e=11910$, respectively. These velocity data have been normalized by the rotation speed of the inner cylinder. The current experimental resolution allowed to capture the velocity vectors as close as $0.35 \mathrm{~mm}$ to the inner and outer cylindrical surfaces. In the case of the laminar flow (circular Couette flow), the theoretical profile of the azimuthal velocity away from the endplates is given by:

$$
u_{\theta}(r)=\operatorname{Ar}+\frac{B}{r}
$$

where $\mathrm{A}=-\omega r_{i}^{2} /\left(r_{o}^{2}-r_{i}^{2}\right)$, and $\mathrm{B}=\omega r_{i}^{2} r_{o}^{2} /\left(r_{o}^{2}-r_{i}^{2}\right)$.

Figure 9. (a) Radial profile of azimuthal velocity of the laminar flow and circular Couette flow profile at $R e=99.2, R a=0$. (b) Mean radial and azimuthal velocity profiles of the turbulent flow at $R e=$ $11910, R a=0$. The measurements were all taken at an axial height of $300 \mathrm{~mm}$ from the bottom.

It can be seen that the experimental results obtained at $R e=99.2$ fit well the theoretical azimuthal velocity profile defined by equation 6 (Figure 9-a). At a much higher value $(R e=11910)$, the flow has, besides a significant azimuthal component, a small radial component (Figure 9-b). The azimuthal velocity is almost constant in the bulk zone and presents sharp gradients in the boundary layers near the cylindrical walls.

\section{E. Torque measurements}

We have measured the torque using the LabVIEW program, which is connected directly to the torque-meter. The obtained values were dimensionalized as follows: $G=T / 2 \pi \rho v^{2} \mathrm{~L}$. In 
the laminar isothermal Couette flow, the dimensionless torque is given by $G_{\text {lam }}=$ $2 \eta R e /\left[(1+\eta)(1-\eta)^{2}\right]$. Following Dubrulle and Hersant ${ }^{67}$, and Ekchardt et al ${ }^{12}$, we converted the dimensionless torque into the pseudo Nusselt number $N u_{\omega}=G / G_{\text {lam }}$ which is the measure of the radial transport of the angular velocity $\omega=u_{\varphi} / r$. The variation of measured $N u_{\omega}$ with the Taylor number is plotted in Figure 10-a. The current experimental data are compared with the correlations provided by Dubrulle and Hersant ${ }^{67}$, Van Gils et al ${ }^{68}$ for the ultimate regime and Wendt ${ }^{69}$ fit for $R e>10^{4}$.

We have also used a compensated plot $N u_{\omega} T^{-0.38}$ against $T a$ to better detect the deviations from the scaling law suggested by Van Gils et al. ${ }^{68}$ (Figure 10-b). We found that for large values of $a\left(\mathrm{Ta}>10^{9}\right)$, our experimental results for isothermal Taylor-Couette flow are comparable with those of van Gils. This represents a good signature of the validity of our torque measurements.

Figure 10. (a) Variation of $N u_{w}$ with Taylor number $T a$. (b) The compensated plot $N u_{w} T a^{-0.38}$ as a function of the Taylor number $T a$ to test the effective scaling.

\section{CONCLUSION}

A new Taylor-Couette system named THETACO has been designed and constructed in Le Havre to investigate the turbulence in thermo-convective flows induced in differentially rotating cylindrical annulus by a radial temperature gradient. The THETACO facility was designed in such a way that the Reynolds number and Rayleigh number can reach values $5 \times 10^{5}$ and $3.36 \times 10^{6}$, respectively. Such a large range of values of the two control parameter allows for investigation of different turbulent states depending on chosen values of Re or Ra. Different optical techniques (LDV, 2D-PIV, and Stereo-PIV) are available for velocity and a new TomoPIV system is under implementation. Torque measurements are feasible using a torquemeter connected to the rotating cylinder. Temperature measurements can be achieved using either thermochromic liquid crystals (TLC) for small temperature differences or LIF for large temperature differences. We have tested the feasibility of most of the techniques and found results which validate the functionality of the THETACO facility. The facility is open for bilateral or international cooperation within specific agreement conditions. 


\section{ACKNOWLEDGEMENTS}

The facility THETACO was built with the financial support from the Normandy Regional Council (grant DIAMECO/APE/HN/2013), the French Ministry of Higher Education, the CNRS (INSIS), the French Ministry of Higher Education and Research (MESR) and the French National Research Agency (ANR) through the program "Investissements d'Avenir (Grant $n^{\circ}$ ANR-10LABX-09-01)", LABEX EMC ${ }^{3}$. H.S. benefited from the financial support from the CPER-FEDER project BIOENGINE.

\section{BIBLIOGRAPHY}

${ }^{1}$ S.G. Huisman, S. Scharnowski, C. Cierpka, C.J. Kähler, D. Lohse, and C. Sun, Physical Review Letters 110, (2013).

${ }^{2}$ M.A. Fardin, C. Perge, and N. Taberlet, Soft Matter 10, 3523 (2014).

${ }^{3}$ C. Coufort-Saudejaud, D. Bouyer, and A. Liné, Chemical Engineering Science 60, 2179 (2005).

${ }^{4}$ M. Vlieghe, C. Coufort-Saudejaud, C. Frances, and A. Liné, AIChE Journal 60, 2389 (2014).

${ }^{5}$ S. Grossmann, D. Lohse, and C. Sun, Physics of Fluids 26, 025114 (2014).

${ }^{6}$ H. Singh, C.A.T. Suazo, and A. Liné, Physical Review E 94, (2016).

${ }^{7}$ O.C. Vadim Travnikov and I. Mutabazi, Acta Astronautica 129, 88 (2016).

${ }^{8}$ P.A. Santiago, R. de C. Giordano, and C.A.T. Suazo, Process Biochemistry 46, 35 (2011).

${ }^{9}$ H. Ji, M. Burin, E. Schartman, and J. Goodman, Nature 444, 343 (2006).

${ }^{10}$ E. Schartman, H. Ji, M.J. Burin, and J. Goodman, Astronomy \& Astrophysics 543, A94 (2012).

${ }^{11}$ S. Mathis, A. Palacios, and J.-P. Zahn, Astronomy \& Astrophysics 425, 243 (2004).

${ }^{12}$ B. Eckhardt, S. Grossmann, and D. Lohse, Journal of Fluid Mechanics 581, 221 (2007).

${ }^{13}$ S. Grossmann, D. Lohse, and C. Sun, Annual Review of Fluid Mechanics 48, 53 (2016).

${ }^{14}$ S.G. Huisman, D.P.M. van Gils, S. Grossmann, C. Sun, and D. Lohse, Physical Review Letters 108, (2012).

${ }^{15}$ S.G. Huisman, R.C.A. van der Veen, C. Sun, and D. Lohse, Nature Communications 5, (2014).

${ }^{16}$ R. Ostilla-Mónico, E.P. van der Poel, R. Verzicco, S. Grossmann, and D. Lohse, Physics of Fluids 26, 015114 (2014).

${ }^{17}$ R. Ostilla-Mónico, R. Verzicco, S. Grossmann, and D. Lohse, Journal of Fluid Mechanics 788, 95 (2016). 
${ }^{18}$ R.A. Verschoof, D. Bakhuis, P.A. Bullee, S.G. Huisman, C. Sun, and D. Lohse, International Journal of Multiphase Flow 105, 264 (2018).

${ }^{19}$ X. Zhu, R. Verzicco, and D. Lohse, Journal of Fluid Mechanics 812, 279 (2017).

${ }^{20}$ X. Zhu, R.A. Verschoof, D. Bakhuis, S.G. Huisman, R. Verzicco, C. Sun, and D. Lohse, Nature Physics 14, 417 (2018).

${ }^{21}$ S. Grossmann and D. Lohse, Physics of Fluids 23, 045108 (2011).

22 S. Grossmann and D. Lohse, Physics of Fluids 24, 125103 (2012).

${ }^{23}$ F. Chillà, M. Rastello, S. Chaumat, and B. Castaing, Physics of Fluids 16, 2452 (2004).

${ }^{24}$ G. Ahlers, E. Bodenschatz, and X. He, Physical Review Fluids 2, 054603 (2017).

${ }^{25}$ X. He, D. Funfschilling, H. Nobach, E. Bodenschatz, and G. Ahlers, Physical Review Letters 108, 024502 (2012).

${ }^{26}$ X. He, D.P.M. van Gils, E. Bodenschatz, and G. Ahlers, New Journal of Physics 17, 063028 (2015).

${ }^{27}$ P. Wei, S. Weiss, and G. Ahlers, Phys. Rev. Lett. 114, 114506 (2015).

${ }^{28}$ A. Pandey, J.D. Scheel, and J. Schumacher, Nature Communications 9, (2018).

${ }^{29}$ J. Sommeria, Nature 398, 294 (1999).

${ }^{30}$ X. Zhu, V. Mathai, R.J.A.M. Stevens, R. Verzicco, and D. Lohse, Phys. Rev. Lett. 120, 144502 (2018).

${ }^{31}$ F. Tachibana, S. Fukui, and H. Mitsumura, Bulletin of JSME 3, 119 (1960).

${ }^{32}$ M. Fénot, Y. Bertin, E. Dorignac, and G. Lalizel, International Journal of Thermal Sciences 50, 1138 (2011).

${ }^{33}$ R. Kedia, M.L. Hunt, and T. Colonius, J. Heat Transfer 120, 65 (1998).

${ }^{34}$ C. Vivès, International Journal of Heat and Mass Transfer 31, 2047 (1988).

35 A. Bahloul, I. Mutabazi, and A. Ambari, Eur. Phys. J. AP 9, 253 (2000).

${ }^{36}$ R. Guillerm, C. Kang, C. Savaro, V. Lepiller, A. Prigent, K.-S. Yang, and I. Mutabazi, Physics of Fluids 27, 094101 (2015).

${ }^{37}$ H.A. Snyder and S.K.F. Karlsson, The Physics of Fluids 7, 1696 (1964).

${ }^{38}$ M.M. Sorour and J.E.R. Coney, Journal of Mechanical Engineering Science 21, 403 (1979).

${ }^{39}$ K.M. Becker and J. Kaye, J. Heat Transfer 84, 106 (1962).

${ }^{40}$ J. Walowit, S. Tsao, and R.C. DiPrima, J. Appl. Mech 31, 585 (1964).

${ }^{41}$ F.C. Haas and A.H. Nissan, Proceedings of the Royal Society of London. Series A, Mathematical and Physical Sciences 261, 215 (1961).

42 J. Chen and J. Kuo, Physics of Fluids A 2, 1585 (1990).

${ }^{43}$ H.N. Yoshikawa, M. Nagata, and I. Mutabazi, Physics of Fluids 25, 114104 (2013). 
${ }^{44}$ K.S. Ball, B. Farouk, V.C. Dixit, Int. J. Heat Mass Transfer 32, 1517 (1989).

${ }^{45}$ D. Liu, S.-H. Lee, and H.-B. Kim, Fluid Dyn. Res. 42, 065501 (2010).

${ }^{46}$ K.G. Roesner, Archiv of Mechanics, Archiwum Mechaniki Stosowanej 30, 619 (1978).

${ }^{47}$ M. Ali and P.D. Weidman, Journal of Fluid Mechanics 220, 53 (1990).

${ }^{48}$ K.S. Ball and B. Farouk, Physics of Fluids A: Fluid Dynamics 1, 1502 (1989).

${ }^{49}$ V. Lepiller, A. Goharzadeh, A. Prigent, and I. Mutabazi, Eur. Phys. J. B 61, 445 (2008).

${ }^{50}$ V. Lepiller, A. Prigent, F. Dumouchel, and I. Mutabazi, Physics of Fluids 19, 054101 (2007).

${ }^{51}$ D. Liu, I.-S. Kang, J.-E. Cha, and H.-B. Kim, Experimental Thermal and Fluid Science 35, $1282(2011)$.

${ }^{52}$ K. Avila and B. Hof, Review of Scientific Instruments 84, 065106 (2013).

${ }^{53}$ D.P.M. van Gils, G.-W. Bruggert, D.P. Lathrop, C. Sun, and D. Lohse, Review of Scientific Instruments 82, 025105 (2011).

${ }^{54}$ S.G. Huisman, R.C.A. van der Veen, G.-W.H. Bruggert, D. Lohse, and C. Sun, Review of Scientific Instruments 86, 065108 (2015).

${ }^{55}$ S. Merbold, H.J. Brauckmann, and C. Egbers, Physical Review E 87, (2013).

${ }^{56}$ M.S. Paoletti, D.P.M. van Gils, B. Dubrulle, C. Sun, D. Lohse, and D.P. Lathrop, Astronomy \& Astrophysics 547, A64 (2012).

${ }^{57}$ E. Schartman, H. Ji, and M.J. Burin, Rev. Sci. Instrum. 9 (2014).

${ }^{58}$ H. Teng, N. Liu, X. Lu, and B. Khomami, Physics of Fluids 27, 125101 (2015).

${ }^{59}$ E. Tuliszka-Sznitko and K. Kiellczewski, J. Phys.: Conf. Ser. 760, 012035 (2016).

${ }^{60}$ C. Kang, K.-S. Yang, and I. Mutabazi, Journal of Fluid Mechanics 771, 57 (2015).

${ }^{61}$ P.G. Wright, Phys. Educ. 12, 323 (1977).

${ }^{62}$ M.A. Dominguez-Lerma, G. Ahlers, and D.S. Canell, Physics of Fluids 28, 1204 (1985).

${ }^{63}$ J.M. Lopez, F. Marques and M. Avila, Int. J. of Heat and Mass Transfer. 90, 959 (2015).

${ }^{64}$ J.W.Elder, J. Fluid Mech. 23, 77 (1965).

${ }^{65}$ J.W.Elder, J. Fluid Mech. 23, 99 (1965).

${ }^{66}$ A. Volk and C.J. Kähler, Exp Fluids 59, 75 (2018).

${ }^{67}$ B. Dubrulle and F. Hersant, Eur. Phys. J. B 26, 379 (2002).

${ }^{68}$ D.P.M. van Gils, S.G. Huisman, G.-W. Bruggert, C. Sun, and D. Lohse, Physical Review Letters 106, (2011).

${ }^{69}$ F. Wendt, Ing. arch 4, 577 (1933). 


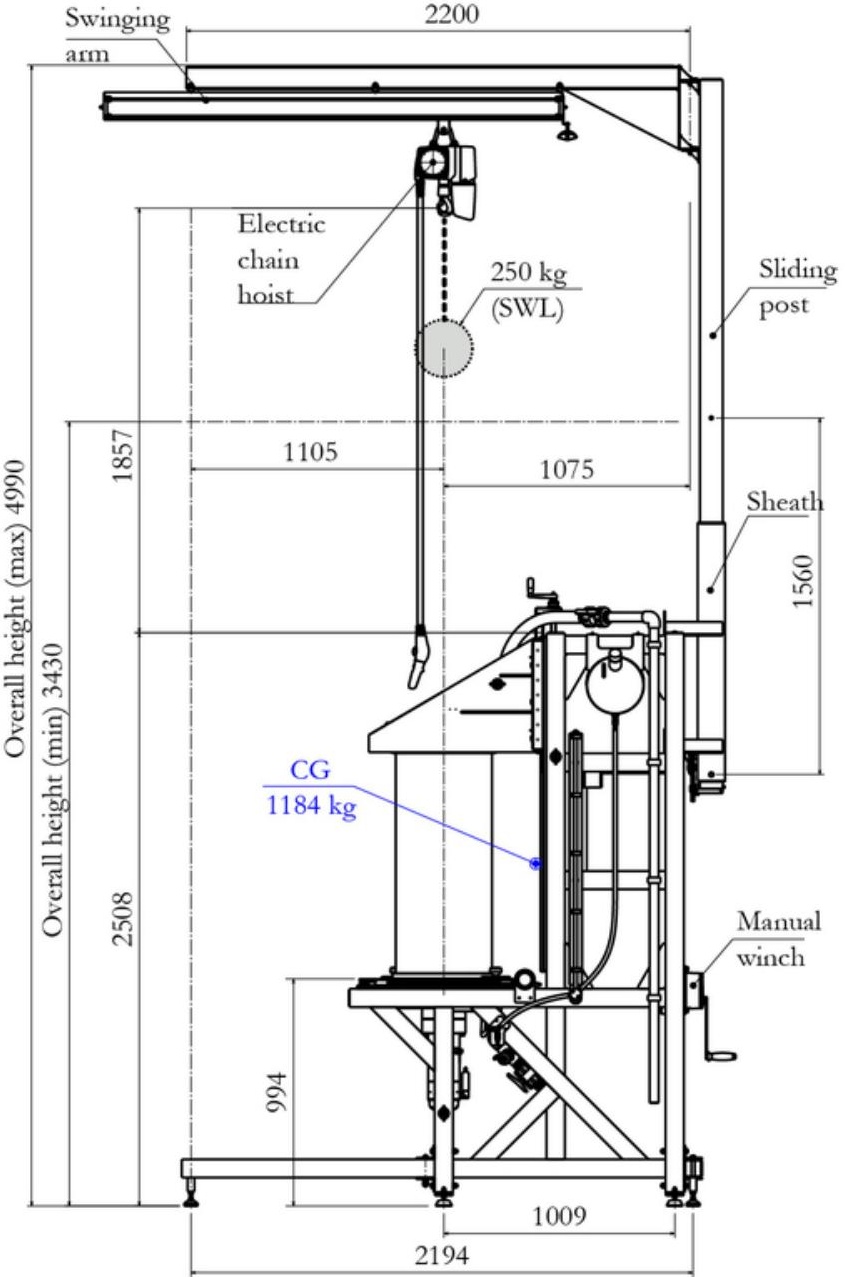


$\Phi 253$ 


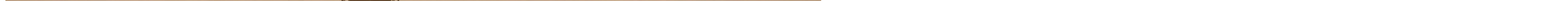




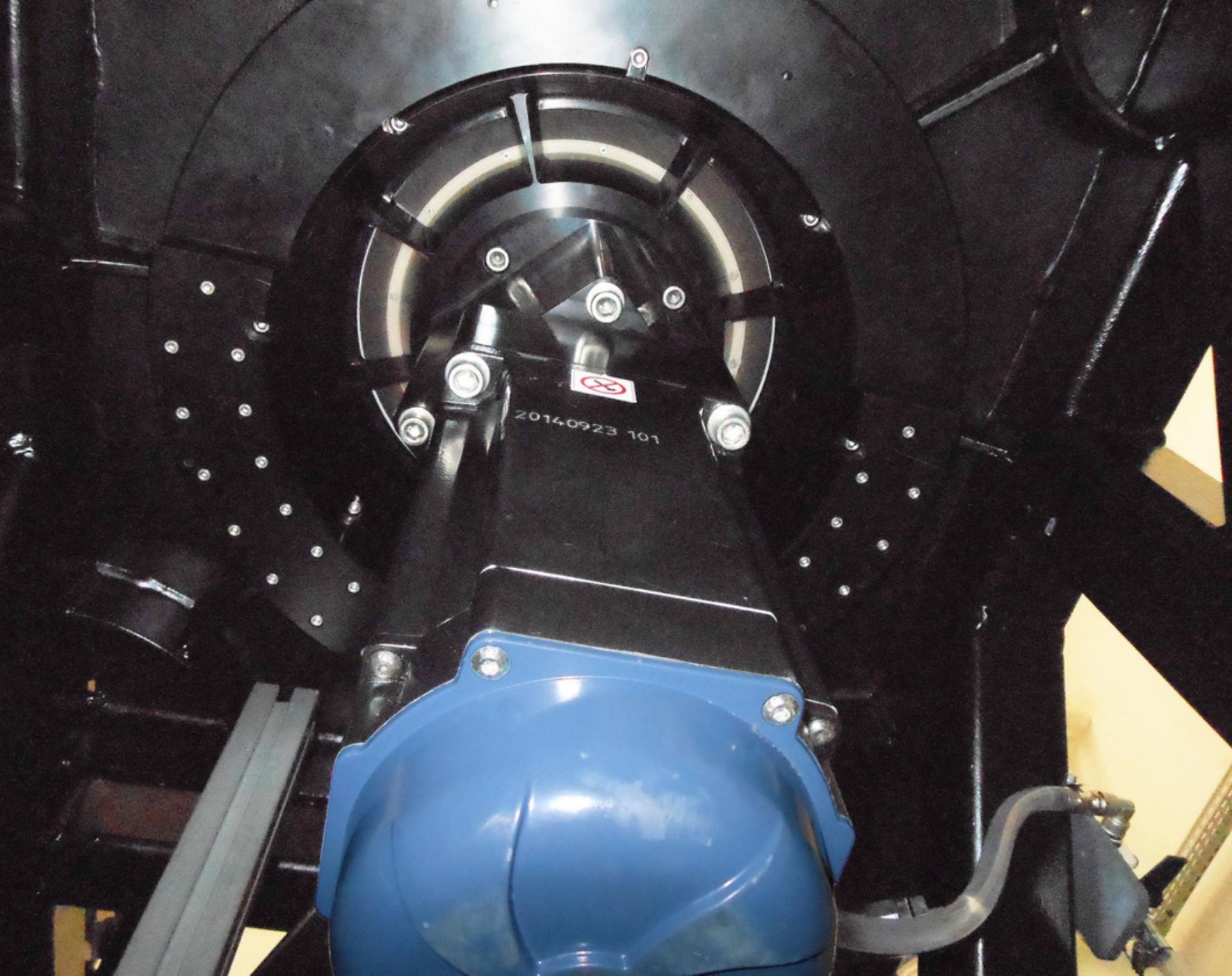




\section{TC SYSTEM}

External Envelop for cooling

Refrigerating Unit

Electrical cabinet

Heating system

6 pyrometers

Motor

Torque-meter

Optical encoder

3 Safety thermocouples in rollers

PC Input Module

Analog Output and $\longleftrightarrow$\begin{tabular}{l:l}
$\hookrightarrow$ & Variator \\
& \\
\hline
\end{tabular}

PID regulators

$\rightarrow \quad$ Logical Module 0/5 v-

Thermocouple Module 


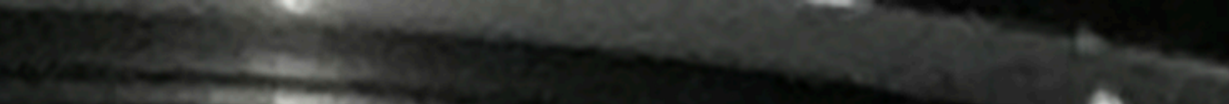




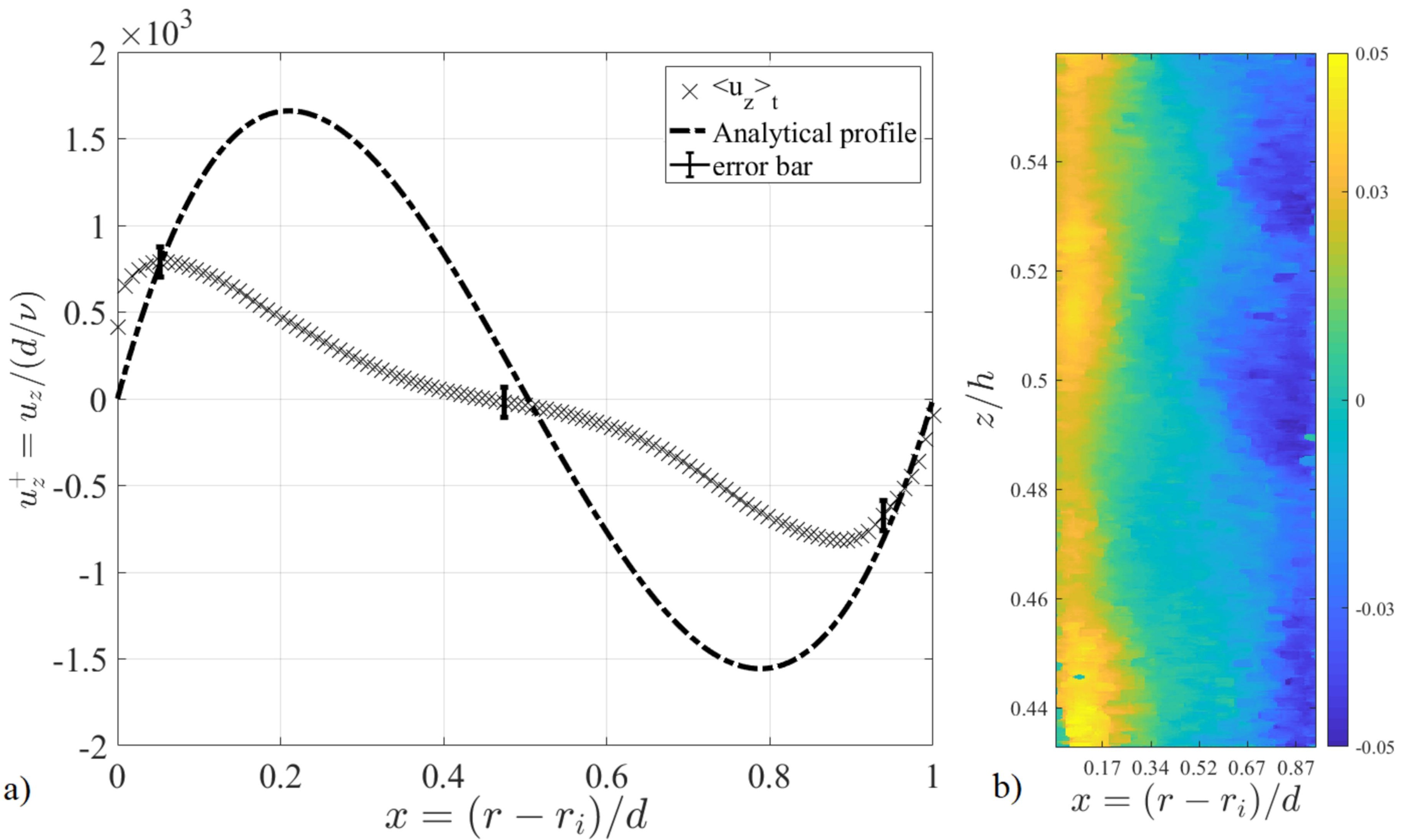




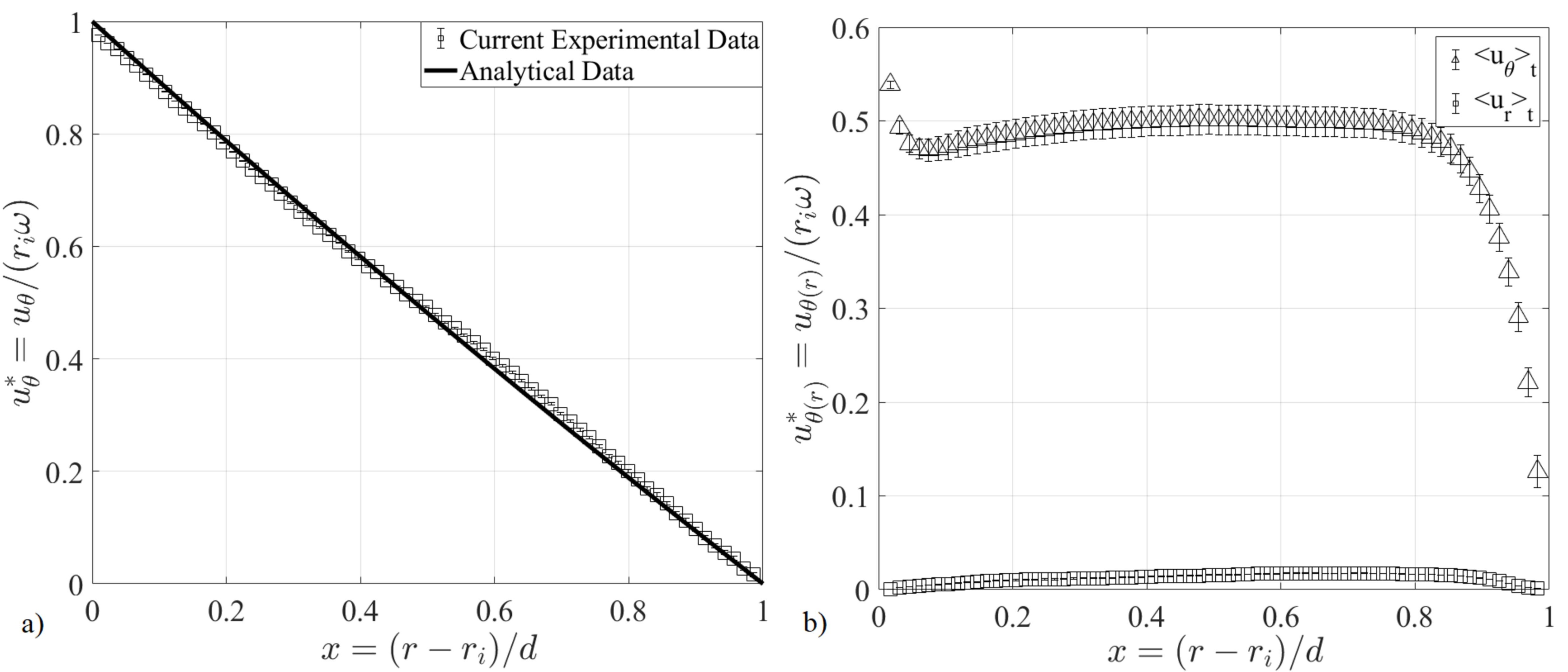




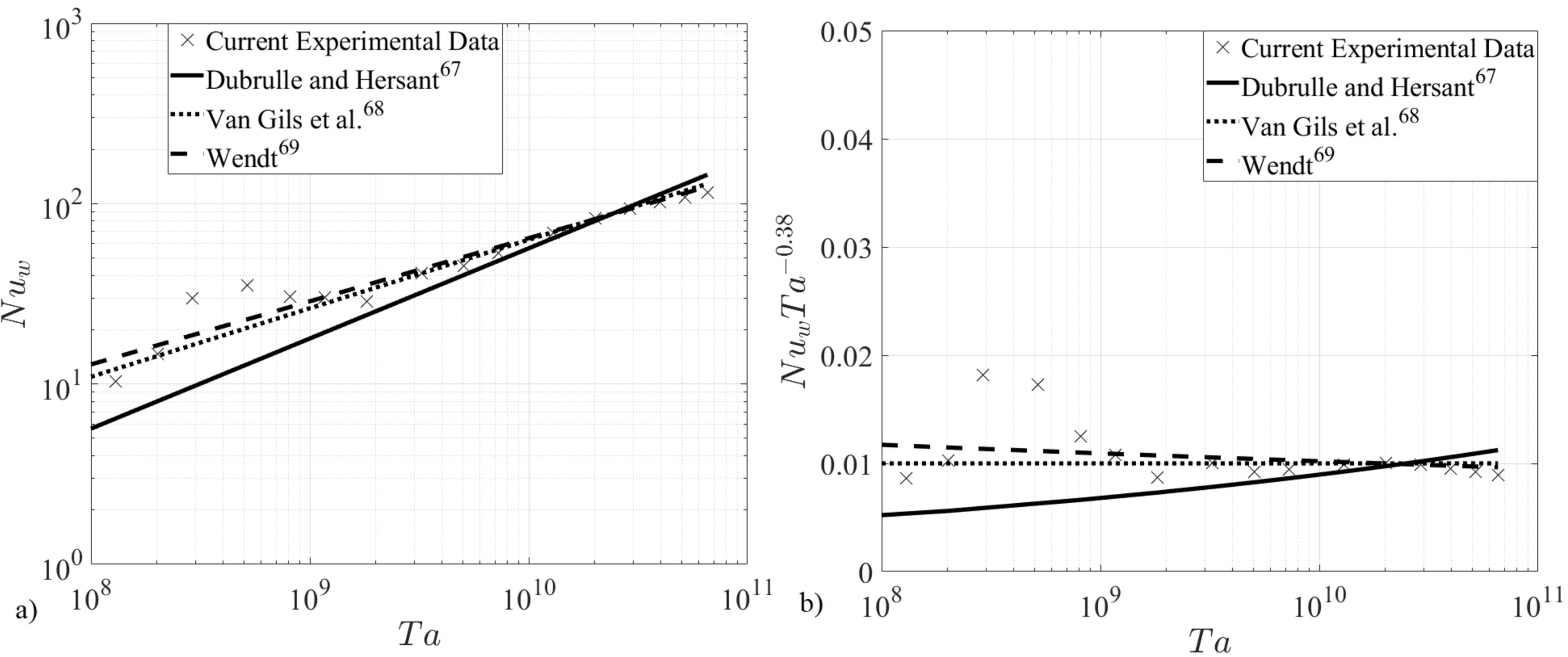

